\title{
Preface of the Special Issue Error Bounds, Regularity, and Nonsmooth Optimization
}

\section{Dedicated to Alexander Kruger on his 65th Birthday}

\section{Regina S. Burachik ${ }^{1} \cdot$ María Josefa Cánovas $^{2} \cdot$ Vera Roshchina $^{3} \cdot$ Claudia Sagastizábal $^{4}$}

Published online: 27 February 2020

(C) Springer Nature B.V. 2020

This special issue pays tribute to the dedication and outstanding work of Professor Alexander Y. Kruger.

Alex Kruger was born in Minsk, the capital city of Belarus, where he defended his PhD thesis under the supervision of Rafail Gabasov and Boris S. Mordukhovich. Currently, he is the Research Director of the Centre for Informatics and Applied Optimization (CIAO), an internationally recognized research centre of the Federation University Australia.

From the very beginning, in the early 80 's, Alex contributed to the theoretical foundations of modern variational analysis and nonsmooth optimization. In 1980 he published two papers (in Russian), coauthored by Boris Mordukhovich, where crucial concepts of variational geometry in Banach spaces were introduced thorugh a dual-space approach and appealing to Fréchet-like constructions. Let us mention the set of $\varepsilon$-normals to a given nonempty subset, giving rise to the basic/limiting normal cone in infinite dimensions, and yielding to remarkable elements such as $\varepsilon$-subgradients and $\varepsilon$-coderivatives. Moreover, those papers introduced the first versions of the extremal principle (appearing there under the name of "generalized Euler equations"), which in a wide sense constitute the variational counterpart (and potential substitute) of the classical separation theorems in the nonconvex setting. Apart from the mentioned papers, Alex elaborated at that time other research works going deeply into extremality notions. Many versions and generalizations of the extremal principle have been developed by different authors until today.

Alex Kruger publications are focussed on extremality, stationarity, transversality, regularity of collections of sets, the error bound property of functions, metric regularity and

Claudia Sagastizábal

sagastiz@unicamp.br

1 School of Information Technology and Mathematical Sciences, University of South Australia, Mawson Lakes, Australia

2 Center of Operations Research, Miguel Hernández University of Elche, Elche, Alicante, Spain

3 School of Mathematics and Statistics, UNSW Sydney, Sydney, Australia

4 IMECC - UNICAMP, Rua Sergio Buarque de Holanda, 651, Campinas, SP 13083-859, Brazil 
subregularity of multifunctions. His works always underline the important role of these concepts in relation to optimality conditions, stability of solutions and convergence of numerical methods.

In addition to the remarkable contributions of Alex's research to the scientific community, his great didactic skills must be emphasized. Particular examples are two works of great educational value: his excellent 2003 survey on the theory of Fréchet subdifferentiation, and his 2009 work "About stationarity and regularity in variational analysis" where a unifying scheme for dealing with extremality, stationarity and regularity in the three typical classes of objects (real-valued functions, collections of sets and multifunctions) is developed.

What may be less noticeable internationally is the enormous work done by Alex within the local Australian community. He has been the Chief Investigator of two consecutive research projects (2011-2015, 2016-2020) funded by the Australian Research Council. In connection with these projects, he trains young researchers, in particular, advising (or coadvising) the doctoral theses of Nguyen Hieu Thao (2015), Hoa Thi Bui (2019), and Nguyen Duy Cuong (on going).

Without any doubt, Alex's leadership and mentoring have been instrumental in setting many talented colleagues and research students on successful career paths; he has introduced outstanding mathematicians to Australia and founded key research activities, including now regular international workshops and seminar series.

Alex is kind, calm, hospitable and reliable. He is a serious person with a great sense of humor, knowledgeable in every aspect of life, and also fair but firm as a teacher. He and his wife Valentina live in Ballarat, a beautiful town in Victoria, Australia, and have two wonderful children.

With this special issue, on the occasion of his 65th birthday, we would like to express our gratitude to Alex Kruger for his contributions to scientific knowledge, to congratulate him for his successful career, and to extend our sincere greetings to his family.

The volume is composed by the following works.

- The paper by Nguyen Hieu Thao, Hoa Thi Bui, Nguyen Duy Cuong and Michel Verhaegen is focussed on the notions of intrinsic transversality and subtransversality in Hilbert spaces. The new bounds and relations obtained by the authors allow to unify and refine existing results, addressing open questions from the literature. Much attention is paid to contextualising the work within other developments in the field and key applications.

- Nguyen Dinh, Miguel A. Goberna and Michel Volle study a new notion of robust sum that leads to duality characterisations and $\varepsilon$-subdifferentiation results for an infinite family of functions defined on a locally convex space.

- Huynh Van Ngai, Nguyen Huu Tron, NguyenVan Vu, and Michel Théra introduce and characterize a new general pseudo subregularity property which formally unifies some important nonlinear (sub)regularity models in the literature.

- The work coauthored by Olga I. Kostyukova, Tatiana V. Tchemisova, and Olga S. Dudina discusses new optimality criteria for linear copositive problems, which do not require the fulfillment of any additional conditions (as constraint qualications), by appealing to the notion of immobile index set.

- Asen L. Dontchev's contribution takes a fresh look at the Bartle-Graves theorem pointing out the main differences with the standard implicit function theorem, and presents a set-valued generalization of this result. He also gives applications of this theorem to variational inequalities and differential inclusions.

- Jean-Pierre Crouzeix, Nadezda Sukhorukova and Julien Ugon provide a better understanding of the alternance property occurring in Chebyshev polynomial approximation 
and continuous piecewise polynomial approximation problems. They prove that alternating sequences of any continuous function are finite in any given segment and then propose an original approach to obtain new proofs of the well known necessary and sufficient optimality conditions. Then, they use their results to develop new local optimality conditions for free knot polynomial spline approximation.

- Matúš Benko, Helmut Gfrerer and Jiří Outrata study a special class of stability parameterized quasi-variational inequalities, giving new sufficient conditions for the isolated calmness property. They also introduce a new concept, the relative Aubin property, that is much weaker than previous assumptions in the literature, typically related to non-degeneracy, and leads to Robinson stability of the considered systems. For the isolated calmness results, not only the sufficient condition is provided under certain assumptions, but the sufficient condition is also proved to be necessary.

- Regina Burachik and Reinier Díaz Millán propose an approximation scheme for solving non-monotone variational inequalities with set-valued operators on finite dimensional spaces. Taking an initial point in the feasible set, the method combines a linesearch with a projection of the initial point onto the intersection of the feasible set with appropriate halfspaces. Three stopping criteria are given and each one turns out to be a sufficient condition for obtaining a solution. A performance comparison with a recent algorithm is given on three examples with point-to-point operators, which show some advantages of the method. An implementation for a point-to-set example is also provided.

- Radek Cibulka, Marián Fabian, and Tomás Roubal prove a statement in the style of Nash-Moser-Ekeland theorem for continuous and directionally differentiable functions between Fréchet-Montel and Fréchet spaces. The finite dimensional case is also dealt and, in particular, results on directional semiregularity are obtained.

As editors, we thank the authors for contributing with excellent material to this special issue. We also want to express our gratitude to the reviewers, whose anonymous job is instrumental for the journal to maintain its high quality standards.

Regina S. Burachik

María Josefa Cánovas

Vera Roshchina

Claudia Sagastizábal

Publisher's Note Springer Nature remains neutral with regard to jurisdictional claims in published maps and institutional affiliations. 